\title{
Prospective study of various methods used in the management of post partum haemorrhage
}

\author{
Jyoti Bindal ${ }^{1, *}$, Sujata Bhargava ${ }^{2}$, Anuradha Gupta ${ }^{3}$ \\ ${ }^{1}$ Professor \& Head, ${ }^{2}$ Associate Professor, ${ }^{3}$ Ex-Resident, Dept. of Obstetrics and Gynecology, ${ }^{1,3}$ GR Medical College, Gwalior, \\ Madhya Pradesh, ${ }^{2}$ Sri Aurboindo Institute of Medical Sciences, Indore, Madhya Pradesh, India
}

*Corresponding Author:

Email: drjyotibindal@bindal.me

\begin{abstract}
Introduction: Mismanagement of postpartum haemorrhage (PPH) is the major reason for the maternal morbidity and mortality throughout the world, highest incidence is found in developing countries.

Aims and objective: To study the various methods used to control PPH in all the deliveries that took place at the study place. Materials and Methods: Prospective study was performed on $402 \mathrm{PPH}$ patients in the Department of Obstetrics and Gynecology, Kamla Raja Hospital, GR Medical College, Gwalior from Sept., 2014 to Aug., 2015. All the patients were divided in to atonic PPH and traumatic PPH. Patient's detailed history including obstetric history, menstrual history and history of drug allergy were recorded in pre-approved excel format. Patients were managed either by catheterization and uterine massage, using uterotonic drugs, uterine packing and surgical intervention such as internal iliac ligation and/or hysterectomay.

Results: Out of total 7977 deliveries, $62.09 \%$ were vaginal and $37.91 \%$ were caesarean section. Out of that 402 (5.03\%) developed PPH. Mean age of study population was $23.3 \pm 1.37$ years. Out of $402 \mathrm{PPH}$ patients, $75 \%$ were having atonic PPH whereas $19.65 \%$ were having traumatic PPH. Most of the patients with atonic PPH were managed by uterotonic drugs (69\%) followed by cathererization with uterine massage (25\%). Most of the patients with traumatic PPH were found to be due to hematoma or tear and were managed by haematoma drainage (58\%) followed by perineal with vaginal repair $(33 \%)$.

Conclusion: Uterotonic drugs were the most commonly used for the management of atonic PPH whereas haematoma drainage was mostly used in patients with traumatic PPH.
\end{abstract}

Keywords: Uterotonic drugs, Post partum haemorrhage, Atonic PPH, Traumatic PPH.

\section{Introduction}

Postpartum hemorrhage (PPH) is the most common cause of maternal morbidity in developing countries like India and also one of the main reasons for the maternal death around the world. ${ }^{1}$

PPH can be defined as the loss of $>500 \mathrm{ml}$ blood after delivery. The prevalence of such blood loss is seen in $18 \%$ of the births. ${ }^{2}$ Blood loss of $>1000 \mathrm{ml}$ is looked as physiologically significant and may lead to hemodynamic instability. ${ }^{1}$

According to latest reports of World Health Organization (WHO), every five minutes, one woman dies during pregnancy and child birth. Out of 529,000 maternal deaths occurring worldwide every year, $136,000(25.7 \%)$ occurs in India. PPH being the most commonly reported complication.

Management of PPH should start from the proper diagnosis of underlying cause, adopting an individualized approach with consideration of suitable choice of option available for the management of PPH such as medical, obstetric, and hematologic intervention. ${ }^{3}$ Hence, present study was performed to study various management methods used for the patients with PPH.

\section{Materials and Methods}

A prospective study was performed on 402 patients with PPH in the Department of Obstetrics and Gynecology, Kamla Raja Hospital, GR Medical
College, Gwalior for one year from Sept., 2014 to Aug., 2015.

All the women admitted with PPH or developed PPH in the hospital after delivery by vaginal or CS were included in the study.

Patient's detailed history including obstetric history, menstrual history and history of drug allergy were recorded in pre-approved excel format. In general examination patient's temperature, pulse, blood pressure, pallor, edema was recorded.

All the patients were divided in to Group I (patients having primary atonic $\mathrm{PPH}$ ) and Group II (patients having traumatic $\mathrm{PPH}$ ).

In Group I after delivery of placenta, uterus was palpated per abdominally and if the uterus was atonic and the blood loss was perceived to be larger than normal, interventions were begun immediately. Volume replacement by crystalloids and blood transfusion was done in each case as per requirement. No standard policy was adopted for these supportive measures. Patients were divided in to four sub groups according to response to management; Group A (patients could be managed by merely catheterization and uterine massage), Group B (Uterotonic drugs needed to control PPH), Group C (uterine packing needed to control $\mathrm{PPH}$ ) and group D (surgical intervention such as internal iliac ligation and/or hysterectomay was needed to control PPH and save life). 
In Group II those patients who have any trauma to the genital tract with or without revealed vaginal bleeding despite a well contracted uterus were included in the group of traumatic PPH.

All the patients of traumatic $\mathrm{PPH}$ were explored under anaesthesia thoroughly and the treatment was individualized in each case. Hematoma drainage, perineal tear repair, compression suture, ligation of arteries or hysterectomies was done as per the need of the case.

All the data was analyzed using IBM SPSS ver. 20 software. $\mathrm{P}$ value $<0.05$ was considered as significant. Results
A total 7977 delivery took place during the study period, out of that $4953(62.09 \%)$ were vaginal and $3024(37.91 \%)$ were caesarean section. The mean age of study population was $23.3 \pm 1.37$ years with ranging from 18 years to 34 years.

Out of 7977 deliveries, $402(5.03 \%)$ developed PPH. Out of 402 patients who had PPH, 308 (76.62\%) were atonic PPH and 94 (23.38\%) were traumatic PPH. Out of $402 \mathrm{PPH}$ patients, $316(78.60 \%)$ were vaginal and $146(36.31 \%)$ were CS.

Out of 316 vaginal deliveries who had PPH, 237 (75\%) were having atonic PPH whereas 79 (19.65\%) were having traumatic PPH. Out of $146 \mathrm{CS}$ deliveries who had PPH, 131 (89.72\%) were having atonic PPH and $15(10.27 \%)$ were having traumatic $\mathrm{PPH}$.

Table 1: Distribution of patients according to different patients characteristic

\begin{tabular}{|c|c|c|c|c|}
\hline \multicolumn{2}{|c|}{ Characteristics } & Atonic PPH & Traumatic PPH & P value \\
\hline \multirow{3}{*}{ Age (years) } & $<20$ & $55(17.8)$ & $19(20.5)$ & \multirow{3}{*}{ NA } \\
\hline & $21-30$ & $202(65.58)$ & $51(54.4)$ & \\
\hline & $>30$ & $51(16.5)$ & $24(25.1)$ & \\
\hline \multirow{3}{*}{$\begin{array}{l}\text { Socioeconomic } \\
\text { status }\end{array}$} & Poor & $199(64.6)$ & $52(55.8)$ & \multirow{3}{*}{$<0.05$} \\
\hline & Average & $66(21.4)$ & $22(23.5)$ & \\
\hline & Good & 43 (13.9) & $20(20.7)$ & \\
\hline \multirow{3}{*}{ Parity } & P1 & $60(19.4)$ & $44(47.14)$ & \multirow{3}{*}{$<0.01$} \\
\hline & $\mathrm{P} 2$ & $73(23.8)$ & $28(29.4)$ & \\
\hline & $\geq \mathrm{P} 3$ & $175(56.8)$ & $22(23.6)$ & \\
\hline \multirow{2}{*}{ ANC status } & Unbooked & $242(79.57)$ & $62(65.71)$ & \multirow{2}{*}{$<0.02$} \\
\hline & Booked & $66(21.33)$ & $32(34.29)$ & \\
\hline \multirow{2}{*}{ Admission type } & Referred & $189(61.36)$ & $66(70)$ & \multirow{2}{*}{$<0.01$} \\
\hline & Direct & $119(39)$ & $28(30)$ & \\
\hline \multirow{2}{*}{ Anemia* } & Mild to moderate & $264(85.71)$ & $64(68.5)$ & \multirow{2}{*}{0.02} \\
\hline & Severe & $44(14.29)$ & $30(31.42)$ & \\
\hline
\end{tabular}

Data is expressed as no of patients (\%), *Mild to moderate anemia (haemoglobin 7 to 10 gm\%), severe anemia (haemoglobin <7 gm \%), PPH; post partum haemorrhage

Out of 308 patients who had atonic PPH, 163 (52.92\%), 105 (34.09\%) and 40 (12.98\%) patients had blood loss of $<1000 \mathrm{ml}$, between $1000-1500 \mathrm{ml}$ and $>1500 \mathrm{ml}$ respectively whereas out of 94 patients with traumatic PPH, 45 (48.05\%), 38 (40\%) and $11(12.85 \%)$ patients had blood loss of <1000 ml, between 1000-1500 $\mathrm{ml}$ and >1500 $\mathrm{ml}$ respectively.

In atonic PPH, $254(82.5 \%)$ responded to crystalloid only while $54(17.5 \%)$ responded to crystalloid plus colloids resuscitation. Most of the Patients with traumatic PPH responded to combination of fluid resuscitation [65 $(69 \%)]$ rather than crystalloid only [29 (31\%)].

\section{Graph 1: Management protocol for atonic PPH}






\author{
$\square$ Mechanical (Catheterization + \\ uterine massage) $(\mathrm{n}=77)$ \\ - Medical (Uterotonic drugs) \\ $(\mathrm{n}=211)$ \\ $\square$ Noninvasive surgical (uterine \\ packing) $(\mathrm{n}=10)$ \\ $\square$ Surgical (arterial ligation) $(\mathrm{n}=4)$ \\ $\square$ Surgical (hysterectomy) $(\mathrm{n}=6)$
}

\title{
Graph 2: Management protocol for traumatic PPH
}



\author{
Haematoma drainage $(\mathrm{n}=53)$ \\ - Perineal+ vaginal tear repair \\ $(\mathrm{n}=29)$ \\ Cervical tear repair $(\mathrm{n}=8)$ \\ - Broad ligament haematoma and \\ internal iliac artery ligation \\ $(\mathrm{n}=4)$ \\ - Hystrectomay $(\mathrm{n}=0)$
}

\section{Discussion}

Proper management of PPH should involve the assessment of different risk factors associated with it and the correct diagnosis of the reasons which can worsen hemorrhage. Obstetricians should choose from the available intervention, what best for the individual patient. $^{3}$

In present study PPH was most commonly seen in age group of 21-30 years which is consistence with the study done by Singh et al. ${ }^{5}$ While study done by Bouwmeester et al reported most common age group of more than 30 years. ${ }^{6}$ The possible reason for this may be due to younger age of marriage in our country which increased gravidity and parity at younger age.

A study done by Dongol et al on 60 patients reported that PPH was most commonly found in unbooked case, deliveries which took place at home and in multiparas which is consistence with the present study findings. ${ }^{7}$ But traumatic PPH in present study was most commonly present in women with single parity. Another study done by Singh et al also reported that most of the PPH cases were multiparas, poor, low socioeconomic class, were unbooked and were from the rural area. ${ }^{5}$ Similar data were reported by present study. This reflects deficiency of good antenatal care, illiteracy and ignorance of this kind of population which is also reported by other studies. ${ }^{8,9}$

More et al performed a similar study on 1023 patients and reported $82.76 \%$ were suffering from atonic and $15.52 \%$ were having traumatic $\mathrm{PPH}$ which is consistence with the present study findings. ${ }^{10}$

Singh et al in their study on referral cases of PPH reported that most of the patients with traumatic PPH $(n=120)$ underwent tear repair and haematoma drainage. They also reported that about $6.67 \%$ of traumatic PPH had peripartum hysterectomy because of rupture uterus. In present study also the most common management protocol for traumatic PPH patients was haematoma drainage $(58 \%)$ followed by perineal with vaginal tear repair $(33 \%){ }^{5}$

Different reports have shown that uterine atony is the most common cause of PPH. ${ }^{5,10,11}$ Agents which are helpful in improving uterine tone and uterine smooth muscle contractility are reported to be the choice of management option. World Health Organization (WHO) has recommended the use of prophylactic 
uterotonic drugs during the $3^{\text {rd }}$ stage of labor along with oxytocin (IM/IV, $10 \mathrm{IU}) .^{12}$

In present study also most of the atonic PPH patients $(69 \%)$ were managed by uterotonic drugs which are consistence with the Singh et al and Ajenifuja et al. ${ }^{5,13}$

Tirumuru et al studied the use of intrauterine balloon tamponade for managing PPH on 50 women reported that $53.5 \%$ were delivered by CS whereas $54 \%$ were delivered vaginally. ${ }^{11}$ which is consistence with the present study data.

Available surgical options for the management of PPH are vessel ligation, uterine compression sutures and hysterectomy. Such procedure expects specialist expertise as they are invasive and involve laparotomy. In present study patient with atonic PPH only $1 \%$ and $2 \%$ undergone for arterial ligation and hysterectomy respectively whereas in traumatic PPH $4 \%$ patients had undergone for internal iliac arterial ligation which is consistent with the study done by Tirumuru et al. ${ }^{11}$

Traumatic PPH can be easily managed if the source of bleeding is discovered mainly in the lower genital tract trauma by the physician. In present study most of the patients were managed by haematoma drainage and vaginal tear repair which is consistence with the study done by Vaidya et al. ${ }^{14}$

Based on the findings, we recommend using uterotonic drugs and haematoma drainage in patients with atonic and traumatic PPH respectively. Small sample size was the main limitation of current study; a large clinical trial is needed to strengthen present study findings.

\section{Conclusion}

Atonic PPH was the most common in present study. Most of the patients with atonic PPH were managed by Uterotonic drugs (69\%) followed by catheterization with uterine massage $(25 \%)$. Uterine packing was performed in only $3 \%$ of atonic PPH patients. Surgical intervention like arterial ligation and hysterectomy was performed in only $1 \%$ and $2 \%$ patients respectively. Most of the patients with traumatic PPH were managed by haematoma drainage $(58 \%)$ followed by perineal with vaginal repair (33\%).

\section{References}

1. Bais JM, Eskes M, Pel M, Bonsel GJ, Bleker OP. Postpartum haemorrhage in nulliparous women: incidence and risk factors in low and high risk women. A Dutch population-based cohort study on standard ( $>$ or $=$ $500 \mathrm{~mL}$ ) and severe ( $>$ or $=1000 \mathrm{~mL}$ ) postpartum haemorrhage. Eur J Obstet Gynecol Reprod Biol 2004;115:166-72.

2. Elbourne DR, Prendiville WJ, Carroli G, Wood J, McDonald S. Prophylactic use of oxytocin in the third stage of labour. Cochrane Database Syst Rev 2001;(4):CD001808.

3. Pavord S, Maybury H. How I treat postpartum hemorrhage. Blood 2015;125 (18):2759-70.
4. Every five minutes, one pregnant woman dies in India: WHO. Hindustan Times. [Internet]. 2017 June 17. Available from: http://www.hindustantimes.com/indianews/every-five-minutes-one-pregnant-woman-dies-inindia-who/story-xoyVjjSEs2oYATHgvfOsoO.html.

5. Singh N, Pandey K, Sharma B, Mehta G, Chandanan A. A Study of Referral Cases of Post Partum HemorrhageStill an Obstetrical Tragedy which is Largely Preventable. Paripex - Indian Journal of Research 2014;3 (6):149-52.

6. Bouwmeester FW, Bolte AC, Van Geijn HP. Pharmacological and surgical management for primary postpartum hemorrhage. Curr Pharma Des 2005;11:75973.

7. Dongol AS, Shrestha A, Chawla CD. Post partum haemorrhage: prevalence, morbidity and management pattern in Dhulikhel Hospital. Kathmandu Univ Med J (KUMJ) 2010 Apr-Jun;8(30):212-5.

8. Subtil D, Somme A, Ardiet E, Deret-Mosser S. Postportum hemorrhage: frequency, consequences in terms of health status, and risk factors before delivery. J Gynecol Obstet Biol Reprod 2004;33(Suppl 4):9-16.

9. Wasim T, Majrooh A, Siddiq S. Maternal Mortality- One year review at Lahore General Hospital. Pak Postgrad Med J 2001;12:113-8.

10. More N, Vaidya S. A Prospective Clinical Study of Postpartum Haemorrhage in Rural Population. Journal of Forensic Medicine, Science and Law 2013;22(1):1-4.

11. Tirumuru S, Saba S, Morsi H, Muammar B. Intrauterine balloon tamponade in the management of severe postpartum hemorrhage: A case series from a busy UK district general hospital. Open Journal of Obstetrics and Gynecology 2013;3:131-6.

12. World Health Organization. WHO recommendations for the prevention and treatment of postpartum hemorrhage. (2012). Global guidelines for prevention and treatment.

13. Ajenifuja KO, Adepiti CA, Ogunniyi SO. Post partum haemorrhage in a teaching hospital in Nigeria: a 5-year Experience. African Health Sciences 2010;10(1):71-4.

14. Vaidya DA, , Kadam S, Rachkonda L, Mande SA, Shiradkar S. Traumatic Post Partum Haemorrhage (PPH) with Dilutional Coagulopathy: A Near Miss Situation. International Journal of Recent Trends in Science and Technology 2013;8(2):132-3. 